\title{
Investigating d-cycloserine as a potential pharmacological enhancer of an emotional bias-learning procedure
}

Marcella L. Woud ${ }^{1}$, Simon E. Blackwell ${ }^{1}$, Susann Steudte-Schmiedgen ${ }^{3}$, Michael Browning ${ }^{4}$, Emily A. Holmes ${ }^{5}$, Catherine Harmer ${ }^{4}$, Jürgen Margraf ${ }^{1}$, \& Andrea Reinecke ${ }^{4}$

1. Mental Health Research and Treatment Center, Department of Psychology, RuhrUniversität Bochum, Massenbergstrasse 9-13, 44787 Bochum, Germany

3. Department of Psychology, Technische Universität Dresden, Zellescher Weg 19, 01062 Dresden, Germany

4. Warneford Hospital, Department of Psychiatry, University of Oxford, Warneford Lane, Oxford OX3 7JX, United Kingdom, UK

5. Department for Clinical Neuroscience, Karolinska Institutet, Solnavägen 1, Solna Alfred Nobels Allé 8, Huddinge, 17177 Stockholm, Sweden

Corresponding author: Marcella L. Woud, Mental Health Research and Treatment Center, Department of Psychology, Ruhr-Universität Bochum, Massenbergstrasse 9-13, 44787 Bochum, Germany. E-mail: marcella.woud@rub.de, phone: +49 (0)234 32 - 21502, fax: +49 (0)234 $32-14369$. 


\begin{abstract}
The partial NMDA receptor agonist d-cycloserine (DCS) may enhance psychological therapies. However, its exact mechanism of action is still being investigated. Cognitive Bias Modification (CBM) techniques allow isolation of cognitive processes and thus investigation of how they may be affected by DCS. We used a CBM paradigm targeting appraisals of a stressful event (CBM-App) to investigate whether DCS enhanced the modification of appraisal, and whether it caused greater reduction in indices of psychopathology. Participants received either 250mg of DCS $(n=19)$ or placebo $(n=19)$. As a stressor task, participants recalled a negative life event, followed by positive CBM-App training. Before and after CBM-App, appraisals and indices of psychopathology related to the stressor were assessed. CBM-App successfully modified appraisals, but DCS did not affect appraisals post-training. There were no post-training group differences in frequency of intrusions. Interestingly, DCS led to a greater reduction in distress and impact on state mood from recalling the event, and lower distress post-training was associated with fewer intrusions. Therefore, DCS may affect emotional reactivity to recalling a negative event when combined with induction of a positive appraisal style, but via a mechanism other than enhanced learning of the appraisal style.
\end{abstract}

Keywords: d-cycloserine; emotional learning; appraisal; emotional reactivity 
Pharmacological cognitive enhancers are promising candidates for improving the efficacy of cognitive behavioural treatment (CBT) for anxiety disorders (for a meta-analysis and review, see Bontempo, Panza, \& Bloch, 2012; Hofmann, Wu, \& Boettcher, 2013; Mataix-Cols et al., 2017; Otto et al., 2015). One such potential enhancer is D-cycloserine (DCS). DCS targets Nmethyl-D-aspartate receptors (NMDAR) by binding with their glycine binding sites, enhancing NMDA receptor-mediated activation. As a result, synaptic plasticity is increased, which is considered a key cellular mechanism involved in emotional, associative learning (e.g., Izquierdo et al., 2006; Sotres-Bayon, Bush, \& LeDoux, 2007). On the assumption that associative, emotional learning is the basis of change in CBT, a number of studies have investigated whether enhancement of neuroplasticity via DCS could have beneficial effects in a therapeutic context, enhancing or accelerating CBT treatment effects. While some findings have been promising, for example in social phobia (Hofmann et al., 2006; Gustaella et al., 2008) or panic disorder (Otto et al., 2010), other studies failed to find a direct, enhancing effect of DCS on treatment outcome, for example in posttraumatic stress disorder (PTSD; de Kleine, Hendriks, Kusters, Broekman, \& van Minnen, 2012) or obsessive compulsive disorder (Kushner et al., 2007).

The reasons for these inconsistent findings are not yet well understood. Accordingly, the present study aimed to further advance our understanding of the mechanisms underlying the effects of DCS on emotional learning. To do this, we took an experimental medicine approach, using a controlled laboratory setting to investigate effects on analogue posttraumatic stress symptoms (i.e., experiences, such as intrusive memories of a distressing or stressful event, assumed to reflect a transient, milder version of PTSD symptoms, such as intrusive memories of trauma; see e.g., James et al., 2016). We used an emotional learning paradigm previously investigated in the context of analogue posttraumatic stress (Woud, Holmes, Postma, Dalgleish, \& Mackintosh, 2012; Woud, Postma, Holmes, \& Mackintosh, 
2013), namely Cognitive Bias Modification - Appraisal (CBM-App) training (for a review on CBM in PTSD, see Woud, Verwoerd, \& Krans, 2017). CBM paradigms involve simple computerized training procedures during which participants are exposed to an experimentally established contingency between a disorder-relevant stimulus and a response, i.e., participants are trained to respond in a systematically biased manner (Koster, Fox, \& MacLeod, 2009). In the context of CBM-App, participants are trained to adopt a positive or negative appraisal style, e.g., towards an analogue stressful event. During training, participants are presented with a series of ambiguous, reappraisal-related scripts. Each script is followed by a word fragment participants are required to complete. Completing the fragment then produces an outcome which is consistent with a functional or dysfunctional appraisal of the script. Studies by Woud et al. showed that, compared to induction of a negative appraisal style, training a positive appraisal style induced more adaptive appraisals and reduced analogue posttraumatic stress symptoms (i.e., such as intrusions over a one-week period (for similar results, see e.g., Cheung \& Bryant, 2017; Schartau, Dalgleish, \& Dunn, 2009). From a mechanisms perspective, the learning that takes place during CBM paradigms such as CBM-App can be conceptualized as emotional associative learning, and more specifically counter-conditioning (cf. Hertel \& Mathews, 2011), in that a stimulus (ambiguous script) potentially signalling a negative outcome (dysfunctional appraisal) is repeatedly paired instead with a positive outcome (functional appraisal), leading to formation of new associations. As such, the action of DCS on NMDA receptors could be expected to enhance associative learning during CBMApp and thus effects of the training.

Investigating the effects of DCS on emotional learning by means of a single session learning paradigm provides at least three advantages. First, many psychological disorders are complex, and the 'learning' during standard treatments such as CBT may also be complex and multi-faceted. Thus, learning effects during treatment may be more difficult to establish. 
Hence, studying the effects of DCS by using a single, distressing event, and focusing on one dysfunctional process, i.e., appraisals, might provide a more efficient means for assessing specific mechanistic processes than is possible with trials of complex interventions. Second, evidence suggests that the effects of DCS are most obvious with a small number of treatment sessions (Otto et al., 2015). Following this, a single learning session may have most potential to reveal DCS's effects. Third, according to Hofmann et al. (2013), learning needs to be established before effects of DCS can be expected, a premise that is not always given during treatment. Interpretation-based CBM procedures robustly revealed training-congruent changes in interpretation (Koster \& Bernstein, 2015; Woud \& Becker, 2014), making this paradigm a promising candidate. To the best of our knowledge, there is only one other study that examined the effects of DCS on a CBM procedure, namely the effects of attention training on trait anxiety (Behar, McHugh, Peckham, \& Otto, 2010). Results demonstrated that both the DCS and placebo group showed less attentional bias post-training. However, this effect was stronger in the DCS compared to the placebo group. Interestingly, DCS did not have an effect on (emotional) stress tasks.

The aim of the present double-blind, placebo-controlled, randomized study was to examine the effects of DCS on affective appraisal and indices of psychopathology following a laboratory stressor. Therefore, one group of participants received a single dose of DCS (250mg), while the other group received a placebo. As a stressor task, participants were instructed to recall a negative life event. This was followed by CBM-App training positive appraisals related to the event. We expected that DCS, compared to placebo, would facilitate emotional learning during the CBM-App training task. Specifically, we expected that participants who received DCS, compared to those who received placebo, would show less dysfunctional appraisals pre-post CBM-App training and at follow-up (assessed via the Encoding Recognition Task, ERT, and the Posttraumatic Cognition Inventory, PTCI). 
Further, we expected that participants receiving DCS, compared to those receiving placebo, would experience fewer intrusions and less intrusion distress post-training (i.e., in-session intrusion questionnaire) and at 24h follow-up (i.e., intrusion diary and intrusions subscale of the Impact of Event Scale - Revised, IES-R). Changes in mood and event-distress were assessed pre-post recall of the negative life event and served as a manipulation check for the impact of recalling the event. Finally, correlational analyses were conducted to examine whether (changes in) appraisals were associated with (changes in) indices of psychopathology, immediately after training and over a 24h period.

\section{Methods}

Participants

Thirty-eight healthy participants (18 female, age range 18-35 years) enrolled in the study after having given written informed consent. Inclusion criteria were: no severe physical illness, no history of neurological or psychological disorder as assessed using the Structured Clinical Interview for DSM-IV Axis I Disorders SCID-CV (First, Spitzer, Gibbon, \& Williams, 1996), no first-degree family member with a history of a severe psychiatric disease, no CNS-active medication or medication with cycloserine, ethionamide, or isoniazid during the last 6 weeks, a body mass index (BMI) between $18-30 \mathrm{~kg} / \mathrm{m}^{2}$, non- or light-smoking $(<5$ cigarettes a day), fluent English skills. Female participants were neither pregnant nor breastfeeding. All participants were included in the analysis. Demographic details and baseline measures included age, years of education, Body Mass Index (BMI), Beck Depression Inventory II (Beck et al., 1996), State Trait Anxiety Inventory - Trait / State (Spielberger et al., 1983), Eysenck Personality Questionnaire - Neuroticism (Eysenck \& Eysenck, 1994); Trauma History Checklist (THC; Holmes et. al., 2004); Posttraumatic Cognitions Inventory baseline (PTCI; Foa et al., 1999), sex, type of employment, ethnicity, first language. The 
study was approved by the Oxford University Medical Sciences research ethics committee (approval number: MSD-IDREC-C3-2014-09), and participants provided written informed consent to take part in the study.

Recall negative life event

To provide a distressing event as a target for the CBM-App training, participants were asked to identify a negative life event (for a similar procedure, see Santa Maria, Reichert, Hummel, \& Ehring, 2012). Before the first full recall of the negative life event, participants were given an information sheet to explain the task requirements. Participants were asked to identify an event that had happened in the past 5 years (if possible), which caused distress at the time that persisted to some degree to the time of experimental testing. Participants were asked to continue to keep the same event in mind throughout the course of their participation in the study. During the first recall, participants were asked bring to mind the most distressing moment of the event and to provide a brief, written description of that moment. Next, participants were instructed to imagine themselves in that moment for 30 seconds. Participants were asked to close their eyes and to imagine the moment as vividly as possible, as if they were experiencing it again, with all images and emotions. Distress caused by recalling the event was indexed by a rating of distress in the present moment ("How distressing is this moment right now"), from 0 (not at all) to 10 (extremely). In addition, participants rated the moment's past distress and vividness, and how detailed their memory was, using an 11-point Likert rating ranging from 0 (not at all) to 10 (extremely) (see Supplements). The second recall deviated only in one aspect from the first recall. That is, participants did not write a summary of the distressing moment but were provided with the summary they wrote during the first recall and were asked to read it silently to themselves. Cognitive Bias Modification - Appraisal (CBM-App) 
The CBM-App training followed the same procedure as the positive training condition in previous studies (Woud et al., 2012; Woud et al., 2013), with the only difference being that the training was anchored to a negative life event rather than a film: Participants were instructed to think back to their specified negative life-event when completing the training. The training task itself comprised processing a series of reappraisal-related scripts that appeared to participants as a sentence completion task. Each script ended with a word fragment such that the meaning of the script remained ambiguous. It was participant's task to finish each script by completing the word fragment. Word fragments were designed so that only one possible solution could complete the script's meaning. In this context, this meant that the completed word fragments produced an outcome consistent with a functional appraisal of the script. Scripts were based on items of the PTCI Self subscale (Foa et al., 1999). For example, "trusting oneself to act appropriately in future” was adapted as follows: 'In a crisis, I predict my responses will be h-lpf-l' (resolved as 'helpful'). Thirty-two of the scripts were followed by a simple yes/no question to test ongoing comprehension (for the example above: 'Do you believe you will be able to respond in a useful way when there is a crisis?'). The training comprised 72 training and 8 neutral filler scripts (presented in blocks of 10). Blocks were presented in the same order for each participant but the sentence order within each block was randomized. A trial was as follows: The script first appeared on the screen without the word fragment. Participants were instructed to press the 'advance' key when they had read the script. After that, the text disappeared, revealing the word fragment. Participants then typed the first missing letter of the fragment as quickly as possible, and the completed correct word appeared on the screen. Next, either a comprehension question or a new script was presented. Appraisal assessment 
Success of the training was assessed primarily via a two-phase Encoding-Recognition Task (ERT; Mathews \& Mackintosh, 2000, for details, see Woud et al., 2012; 2013). During the encoding-phase, participants read 10 novel ambiguous scripts in random order. Scripts started with a title and remained ambiguous. Participants were asked to imagine themselves vividly in the described situation. In the recognition-phase, the 10 encoding-phase titles were presented again, followed by a set of 4 related sentences. By means of a 4-point Likert scale, participants rated how close in meaning each sentence was to the original script of that title. There were two target sentences, representing a possible positive and negative interpretation of the original script, and there were two foil sentences, representing a general positive and negative meaning that did not resolve the script's ambiguity. A bias index was calculated by subtracting the mean recognition rating for negative targets from that for positive targets, such that a positive score indicates a relative bias for endorsing positive over negative interpretations. There were two sets of scripts, the order of which was counterbalanced across participants.

As a further measure of change in appraisal, participants completed the Posttraumatic Cognition Inventory (PTCI; Foa et al., 1999). The PTCI is a self-report measure including 36 statements assessing appraisals surrounding distressing or traumatic experiences. It contains three subscales: negative cognitions about Self (21 items), negative cognitions about the World (7 items) and Self-Blame (5 items). Each item is rated using a 7-point Likert scale ranging from 1 (totally disagree) to 7 (totally agree). The instructions were modified in that participants were asked to think back of their own negative life event (as opposed to a trauma) when completing the PTCI.

\section{Mood}

Four mood states were assessed over the course of the study (happiness, depression, anger, anxiety, see Woud et al., 2012, 2013) using 11-point scales ranging from 0 (not at all) 
to 10 (extremely). Scores across the 4 scales were averaged (happiness reverse scored) to provide a single mood index.

Negative involuntary memory assessment

In-session intrusion questionnaire. The number and quality of intrusions related to the negative life event were assessed with an adapted version of the intrusion diary used by Woud et al. (2012; 2013). The diary was presented as a questionnaire in order to assess intrusions during the session. On the questionnaire, intrusions were defined as "any memory of the negative life event (or part of it) that appears apparently spontaneously in mind. Do not include any memories that you deliberately or consciously bring to mind.” Participants were also instructed that intrusions could be experienced as an image, verbal thought, or combination of both, and were asked to specify this for each intrusion they recorded. Further, participants were asked to record how distressing each intrusion was on a rating scale ranging from 0 (not at all) to 100 (extremely) and to provide an overall vividness rating of their intrusions from 0 (not all vivid) to 100 (extremely).

24h intrusion diary. To assess intrusions that occurred during the 24h after testing, participants were provided with an intrusion diary. This had the format of the in-session intrusion questionnaire used during the laboratory session, i.e., intrusions were defined and the different types of intrusions were explained. Participants were instructed to record all intrusions immediately after they occurred (whenever possible). If participants had experienced no intrusions during any period they were also asked to make this explicit in the diary.

Intrusion subscale Impact of Events Scale - Revised (IES-R; Weiss \& Marmar, 1997). The IES-R is a self-report measure including 22 items assessing current intrusion, avoidance, and hyperarousal phenomena tied to a stress-inducing event. The instructions were adapted to reflect experiences during the past 24h, linked to the negative life event (as 
opposed to the past week and a traumatic event), and the intrusion subscale was used as an additional measure of intrusive memories.

Procedure

Participants were randomly allocated to a single dose of DCS (250 mg) or a matching placebo capsule (major ingredient: microcrystalline cellulose) in a double-blind, placebocontrolled design. The drug's dose was chosen based on previous studies showing cognitive effects of DCS within a range of 50mg to 500mg (Klumpers et al., 2012; Onur et al., 2010). Participants fasted $2 \mathrm{~h}$ before testing to limit gastrointestinal influences on drug pharmacokinetics, and they were tested 3h after drug administration, the time of estimated peak plasma levels (King Pharmaceutical product information). The drug manufacturer's (King's Pharmaceutical) product information suggests that plasma levels are reached within 3-4h. However, other studies report peak levels to be reached within 1h (van Berckel et al, 1997; Patel et al., 2011). Considering that DCS is thought to have half-life properties of 8h (King’s Pharmaceutical product information) to 15h (Patel et al., 2011), plasma levels were expected to be close to peak levels during testing.

Demographic details and baseline measures were collected prior to administration of drug or placebo, and subjective effects of perceived side-effects of the drug were taken at baseline, drug peak level, and at the end of the session. Prior to the experimental procedures relevant to this manuscript, participants worked on a tactile sensory learning task (unrelated to the current study) ${ }^{1}$, to explore whether DCS affects this form of non-emotional learning relevant to recovery after stroke. Plastic dome gratings with varying groove widths were used for testing and training, and participants indicated whether a grating was presented horizontally or vertically on the glabrous surface of the distal finger pad. Before and after the CBM-App training task, participants completed the appraisal assessments (i.e, ERT and PTCI) and the negative memory recall, with the latter also including the distress ratings. Further, the 
occurrence of intrusive memories of the recalled event was recorded throughout the session via the in-session intrusion questionnaire. Prior to the administration of the questionnaire, participants sat quietly for 2 mins, wearing a chest strap heart rate monitor for the purposes of an approved deception that a resting heart rate was being recorded (for a similar procedure, see Santa Maria et al., 2012). Mood was rated four times: pre-post first recall (pre CBM-App training), and pre-post second recall (post CBM-App training). At the end of the first session, participants received a diary to monitor their intrusions over the following $24 \mathrm{~h}$, after which they returned to the laboratory to complete the follow-up (i.e., IES-R and PTCI). Figure 1 gives a diagrammatic overview of the procedure. The Supplements describes some additional measures (i.e., side effects drug, demand effects, and diary compliance rating) and analyses, and includes a more detailed description of the procedure.

\section{Design and statistical analyses}

The present study used a between-subjects design which included two groups: DCS and placebo. Repeated-measures ANOVAs were conducted to examine changes in appraisal, distress, and mood from pre- to post-training and pre-post recall of the negative life event, respectively. Time x Group interactions indicate the outcomes of interest. If significant, paired sample t-tests were conducted to decompose the interaction. Outcomes at follow-up were compared between-groups tests or non-parametric equivalents in the case of severely skewed distributions or lack of homogeneity between groups. Correlational analyses used Kendall tau indices. Demographic and baseline data and means and standard deviations of all outcome measures are presented in Table 1 and 2, respectively. The supplements provides additional analyses such as questionnaire subscales.

\section{Results}

Baseline data 
There were no statistically significant differences between the groups prior to the CBM training (all ps > .05), except that in the DCS group fewer participants were of Caucasian ethnicity ( $p=.046$, Fisher's exact test).

\section{Appraisal assessment}

Pre-post CBM-App, ERT. The CBM training successfully modified participants' appraisal in the intended direction on the Encoding-Recognition Task (ERT) from pre- to post-training (Time: $F_{1,33}=48.64, \mathrm{p}<.001, \eta^{2}=.60$ ), but this effect did not differ between groups (Group x Time: $\left.F_{1,33}=.046, p=.83, \eta^{2}=.001\right)^{2}$

Pre-post CBM App, PTCI. Results of the Posttraumatic Cognitions Inventory (PTCI) mirrored those of the ERT. That is, participants reported less dysfunctional appraisals posttraining (Time: $F_{1,36}=21.36, p<.001, \eta^{2}=.37$ ), but this was not qualified by group (Group x Time: $\left.F_{1,36}=0.86, p=.36, \eta^{2}=.023\right)$.

Follow-up, PTCI. There was no difference between the two groups in scores on the Posttraumatic Cognitions Inventory (PTCI) at follow-up $t(35)=1.11, p=.28$

\section{Manipulation checks recall negative life event pre-post CBM-App}

Effects on distress. Participants' distress on recalling the event decreased from pre- to post-training (Time: $F_{1,36}=8.94, p=.005, \eta^{2}=.2$ ), and this decrease differed between groups (Group x Time: $F_{1,36}=4.78, p=.035, \eta^{2}=.12$ ). Follow-up t-tests showed that there was a decrease in ratings of distress in the DCS group $(t(18)=3.75, p=.001, d=0.81)$, but not in the placebo group $(t(18)=0.56, p=.59, d=0.09)$ (see Figure 2).

Effects on mood. Participants' mood response to recalling the event (i.e. change in state mood from pre- to post-recall, indicated by the Time factor in the analyses) changed from pre- to post-training, and this change differed per group (Time x Pre-/Post-Training $\mathrm{x}$ Group: $\left.F_{1,36}=5.04, p=.031, \eta^{2}=.12.\right)$. This 3-way interaction was decomposed by 2 additional ANOVAs per group, both revealing a significant Time (i.e., Pre- to Post-recall) x 
Pre-/Post-Training interaction (DCS: $F_{1,18}=23.67, p>.001, \eta^{2}=.57$; Placebo: $F_{1,18}=$ 11.31, $p=.003, \eta^{2}=.39$ ). Paired sample t-tests comparing the pre-training mood changes (i.e., Mood I vs. Mood II) further showed that both groups’ mood became more negative after the first recall of the negative event $(\mathrm{DCS}: t(18)=4.33, p<.001, d=3.79$; Placebo: $t(18)=$ 2.87, $p=.01, d=0.64$ ). When comparing the post-training mood changes (Mood III vs. Mood IV), results showed that the mood of the DCS group became better $(t(18)=2.42, p=.03, d=$ $0.45)$. However, there was no such change in the placebo group $(t(18)=.8, p=.44)$ (see Figure 2).

\section{CBM-App effects on intrusive memories}

In-session intrusion questionnaire. Participants reported fewer intrusive memories in the 2 mins after the negative memory recall post- compared to pre-training (Time: $F_{1,36}=$ 23.06, $p<.001, \eta^{2}=.39$ ). However, this reduction did not differ between the groups (Time $\mathrm{x}$ Group: $F_{1,36}=0.22, p=.65$ ). Due to the small number of intrusions, we did not analyse the data for intrusion distress and vividness.

24h diary. There was no difference between groups in number of intrusive memories recorded in the 24h diary $(U=146, Z=0.81, p=.46$. $)$.

Intrusion subscale IES-R. There was no difference between groups on the Impact of Event Scale - Revised (IES-R) intrusion subscale at follow-up, $U=141, Z=0.93, p=.37$.

\section{Correlational analyses}

First, we examined whether changes in appraisals (ERT and PTCI) were associated with changes in indices of psychopathology (number of intrusions, distress after recall) from pre- to post-training. Contrary to expectations, none of these correlations were significant (all ps $>.05)$. Second, we examined whether distress experienced after the second recall was associated with indices of psychopathology 24h later (i.e., PTCI, diary intrusions, IES-R intrusion subscale). Distress associated with recalling the event post-training correlated with 
diary intrusions $(\tau=.37, p<.01)$ and the IES-R intrusion subscale $(\tau=.52, p<.01)$, showing that a lower level of distress associated with the second (post-training) recall was associated with fewer intrusions over the subsequent $24 \mathrm{~h}$.

\section{Discussion}

The current study investigated whether administration of d-cycloserine (DCS) would facilitate learning during an emotional, associative learning task (CBM-App). We expected that administration of DCS, compared to placebo, would be associated with less dysfunctional appraisals and fewer stress symptoms. Results showed that overall, appraisals were less dysfunctional following CBM-App (as shown by changes on the ERT and PTCI from pre- to post- CBM-App). Contrary to our expectations, there was no greater decrease in dysfunctional appraisals in the DCS group compared to the placebo group. Additionally, the two groups did not differ on appraisals at follow-up (measured by the PTCI). We further did not find the expected group difference on any of the three intrusion measures, that is, the insession intrusion questionnaire administered during the laboratory session, the $24 \mathrm{~h}$ diary, or the intrusions subscale of the IES-R. However, participants in the DCS group showed reduced emotional reactivity to recalling a negative life event, as indexed by both a measure of distress and change in state mood. Finally, correlational analyses showed that less distress after the recall was associated with fewer intrusive memories about the negative event over a 24h period.

There are at least three possible explanations as to why we did not find differential effects of DCS on our CBM learning paradigm. First, it is possible that this reflects a ceiling effect due to general effectiveness of the CBM training. Participants in both groups showed more positive appraisals post-training, suggesting that the training task in itself was sufficient for successful learning and there was not much scope for DCS to further enhance it. Second, 
the Encoding Recognition Task (ERT) may not have been sensitive enough to capture any effects DCS may have had on learning. These suggestions are consistent with other studies showing that DCS does not have an effect on overall learning, but instead leads to differences in more subtle measures of trial-by-trial responding (Scholl et al., 2014, and for similar results, see e.g., Rothbaum et al., 2014). A third potential explanation is that DCS may in fact not have robustly demonstrable effects on emotional learning, or even non-emotional learning (e.g., Günthner et al., 20116; Butler et al., 2015; Cherry, Lenze, \& Lang, 2014). However, there are many (subtle) factors that have to be taken into account when studying the effects of DCS (Hofmann, Otto, Pollack, \& Smits, 2015), including factors such as drug dose, timing, and participants' medication status, or the functional properties of the intervention itself. Hence, it may be that there are definable circumstances in which robust effects of DCS on (emotional) learning could be found.

In the present study, change in appraisals was used as an index for the effect of DCS on learning. Although there was no evidence for an effect of DCS on appraisals, other measures did suggest differential effects: In the DCS but not in the placebo group, participants' distress following the recall of the negative life event decreased from pre- to post-training. Further, the DCS group’s mood improved after the second recall, compared to the mood of the placebo group. To summarize, the DCS group showed reduced emotional reactivity, compared to the placebo group. Given the fact that these findings are secondary, they should be interpreted with caution. Nevertheless, we suggest some potential explanations: One possibility is that following the training, participants in the DCS group appraised their negative life event in a more adaptive manner than the placebo group, which in turn made them less vulnerable to the effects of the recall. However, at this stage it is difficult to know whether these effects are in fact a result of the drug enhancing a subtle beneficial effect of the CBM training on appraisal, or via a direct effect that would also have 
been found in the absence of the CBM-App training. For example, it may be that the reduction in memory distress post-training is an effect of exposure to the memory pretraining, and it is this exposure-learning that DCS enhanced (Hofmann et al., 2013), or that DCS just had a direct effect on mood.

A limitation of the current study is the degree to which the recall task was able to elicit strong emotional reactions and analogue posttraumatic-stress symptoms among participants: Despite the significant worsening in negative mood in both groups after the first recall, participants’ mood was still generally positive. Further, the number of intrusions pretraining were small. Future studies may need to further optimize such analogue trauma manipulations so that in order to obtain robust effects on stress parameters. A second, related, limitation, is that the sample was not selected on the basis of pre-training stress symptoms (although we note that Santa-Maria et al., 2012 also did not use such a selection). Both these issues may have limited our ability to observe beneficial effects of DCS-enhanced learning. The administration of the study procedures after administration of DCS or placebo precluded the use of a potentially more powerful and standardized experimental trauma analogue such as a distressing film (James, Lau-Zhu, Clark, Visser, Hagenaars, \& Holmes, 2016), as DCS may have influenced initial processing and consolidation of the negative event itself. Thus, in this first study we relied on negative memories already experienced by participants. However, future studies could benefit from either using a standardized analogue trauma such as a film prior to DCS administration, or a selected sample of individuals recruited on the basis of having experienced a highly distressing event of which they experience intrusive memories. Follow-up investigations should also to include a DCS-only group in order to assess whether potential benefits of DCS were in fact via enhancing the effects of CBM-App or were instead a direct effect independent of the training. Finally, the study had a relatively short time-frame, and so we do not yet know whether our findings can 
be attributed to a sub-optimal combination of dose and time of administration (Hofmann et al., 2015).

In the present study, by combining a pharmacological enhancer (DCS) with an emotional bias learning paradigm (CBM-App), we aimed to contribute to the growing literature recognising the potential benefits of combining psychological and pharmacological approaches in developing more effective treatments for mental health problems (e.g., Moss et al., 2016; Reinecke \& Harmer, 2014). Our results showed that DCS did not have an effect on our measure of learning, that is, appraisals of a negative life event. However, DCS reduced participants' emotional reactivity in response to recalling a negative life event after participants had completed the learning task. This suggests either that DCS had a direct effect on reducing the emotional response to a negative memory after a minimal reactivation, or that it enhanced the application of the learning (a more positive appraisal style) to the negative memory. These hypotheses provide interesting avenues for future research aiming to enhance psychological therapy outcomes via pharmacological agents. 
DCS in emotional learning

Woud et al.

\section{Acknowledgements}

We are grateful to Lorika Shkreli for her help in preparing the manuscript. 


\section{Funding and conflict of interest}

This research received no specific grant from any funding agency in the public, commercial, or not-for-profit sectors.

Authors MLW, SEB, SSS, JM, and AR report no conflict of interest. MLW is supported by a PostDoctoral scholarship of the Daimler and Benz Foundation (32-12/4) and by a research grant of the Deutsche Forschungsgemeinschaft (DFG; WO 2018 / 2-1). MB is supported by a Clinician Scientist Fellowship from the MRC (MR/N008103/1) and receives consultancy fees from P1vital ltd and Lundbeck. EAH receives a salary from the Karolinska Institutet and receives funding from The Wellcome Trust, the UK NIHR, and the Lupina Foundation. EAH is Associate Editor for Behaviour Research and Therapy and receives an honorarium; she is on the editorial boards of Cognitive Behaviour Therapy and Psychological Science and receives no remuneration. EAH is on the Board of Trustees of the Charity "MQ; transforming mental health" and receives no remuneration for this role. EAH is a member of Clare Hall, Cambridge University and receives a food allowance. EAH has a co-authored book on Imagery in Cognitive Therapy (OUP, 2011), that receives royalties. EAH presents at clinical training workshops on mental imagery and CBT some of which include a fee. EAH is an Honorary Professor of Clinical Psychology at the University of Oxford, Department of Psychiatry and a Visiting Scientist at the MRC Cognition and Brain Sciences Unit, Cambridge UK until 2018 and receives no remuneration for this roles. $\mathrm{CH}$ receives consultancy fees from P1vital ltd and Lundbeck, and grant income from J\&J, UCB, Sunovion, Servier, and Lundbeck.

Materials and data related to this paper can be requested via e-mail via the first author. 


\section{References}

Behar, E., McHugh, R. K., Peckham, A., Otto, M. W. (2010). d-Cycloserine for the augmentation of an attentional training intervention for trait anxiety. Journal of Anxiety Disorders, 24, 440-445. doi:10.1016/j.janxdis.2010.02.009.

Beck, A. T., Steer, R. A., \& Brown, G. K. (1996). Beck Depression Inventory - II Manual (2nd ed.). San Antonio, TX: The Psychological Corporation.

Bontempo, A., Panza, K. E., \& Bloch, M. H. (2012). D-cycloserine augmentation of behavioral therapy for the treatment of anxiety disorders: a meta-analysis. Journal of Clinical Psychiatry, 73(4), 533-537. doi: 10.4088/JCP.11r07356.

Butler, A. J., Kallos, J., Housley, S. N., LaPlaca, M. C., Traynelis, S. F., \& Wolf, S. L. (2015). Randomized, placebo-controlled, double-blind pilot study of D-cycloserine in chronic stroke. Rehabilitation Research and Practice, 2015.

Cherry, K. M., Lenze, E. J., \& Lang, C. E. (2014). Combining d-cycloserine with motor training does not result in improved general motor learning in neurologically intact people or in people with stroke. Journal of Neurophysiology, 111(12), 2516-2524.

Cheung, J., \& Bryant, R. A. (2017). The impact of appraisals on intrusive memories. Journal of Behavior Therapy and Experimental Psychiatry, 54, 108-111.

de Kleine, R. A., Hendriks, G. J., Kusters, W. J., Broekman, T. G., \& van Minnen, A. (2012). A randomized placebo-controlled trial of D-cycloserine to enhance exposure therapy for posttraumatic stress disorder. Biological Psychiatry, 71(11), 962-968. doi: 10.1016/j.biopsych.2012.02.033.

Eysenck, H.J., \& Eysenck, S. B. G. (1994). Manual for the Eysenck Personality Questionnaire (EPQ R Adult). Educational Industrial Testing Service.

First, M. B., Spitzer, R. L., Gibbon M., \& Williams, J. B. W. (1996). Structured Clinical 
Interview for DSM-IV Axis I Disorders, Clinician Version (SCID-CV). Washington, D.C.: American Psychiatric Press, Inc.

Foa, E. B., Ehlers, A., Clark, D. M., Tolin, D. F., \& Orsillo, S. M. (1999). The Posttraumatic Cognitions Inventory (PTCI): Development and validation. Psychological Assessment, 11(3), 303-314. doi: 10.1037/1040-3590.11.3.303

Guastella, A. J., Richardson, R., Lovibond, P. F., Rapee, R. M., Gaston, J. E., Mitchell, P., \& Dadds, M. R. (2008). A randomized controlled trial of D-cycloserine enhancement of exposure therapy for social anxiety disorder. Biological Psychiatry, 63(6), 544-549. doi: 10.1016/j.biopsych.2007.11.011

Günthner, J., Scholl, J., Favaron, E., Harmer, C. J., Johansen-Berg, H., \& Reinecke, A. (2016). The NMDA receptor partial agonist d-cycloserine does not enhance motor learning. Journal of Psychopharmacology, 30(10), 994-999.

Hertel, P. T., \& Mathews, A. (2011). Cognitive bias modification past perspectives, current findings, and future applications. Perspectives on Psychological Science, 6(6), 521536. doi: 10.1177/1745691611421205

Hofmann, S. G., Meuret, A. E., Smits, J. A., Simon, N. M., Pollack, M. H., Eisenmenger, K., ... \& Otto, M. W. (2006). Augmentation of exposure therapy with D-cycloserine for social anxiety disorder. Archives of General Psychiatry, 63(3), 298-304. doi: 10.1001/archpsyc.63.3.298

Hofmann, S. G., Otto, M. W., Pollack, M. H., \& Smits, J. A. (2015). D-cycloserine augmentation of cognitive behavioral therapy for anxiety disorders: an update. Current Psychiatry Reports, 17(1), 1-5. doi: 10.1007/s11920-014-0532-2.

Hofmann, S. G., Wu, J. Q., \& Boettcher, H. (2013). D-Cycloserine as an augmentation strategy for cognitive behavioral therapy of anxiety disorders. Biology of Mood \& Anxiety Disorders, 3(1), 1. doi: 10.1186/2045-5380-3-11. 
Holmes, E. A., Brewin, C. R., \& Hennessy, R. G. (2004). Trauma films, information processing, and intrusive memory development. Journal of Experimental Psychology: General, 133(1), 3-22. doi: 10.1037/0096-3445.133.1.3

Izquierdo, I., Bevilaqua, L. R. M., Rossato, J. I., Bonini, J. S., Medina, J. H., \& Cammarota, M. (2006). Different molecular cascades in different sites of the brain control memory consolidation. Trends in Neurosciences, 29(9), 496-505.

James, E. L., Lau-Zhu, A., Clark, I. A., Visser, R. M., Hagenaars, M. A., \& Holmes, E. A. (2016). The trauma film paradigm as an experimental psychopathology model of psychological trauma: intrusive memories and beyond. Clinical Psychology Review. doi: 10.1016/j.cpr.2016.04.010.

Klumpers, F., Denys, D., Kenemans, J. L., Grillon, C., van der Aart, J., \& Baas, J. M. (2012). Testing the effects of $\Delta 9$-THC and D-cycloserine on extinction of conditioned fear in humans. Journal of Psychopharmacology, 26(4), 471-478.

Koster, E. H., \& Bernstein, A. (2015). Introduction to the special issue on Cognitive bias modification: Taking a step back to move forward? Journal of Behavior Therapy and Experimental Psychiatry, 49, 1-4. doi: 10.1016/j.jbtep.2015.05.006

Koster, E. H., Fox, E., \& MacLeod, C. (2009). Introduction to the special section on cognitive bias modification in emotional disorders. Journal of Abnormal Psychology, 118(1), 1. doi: 10.1037/a0014379

Kushner, M. G., Kim, S. W., Donahue, C., Thuras, P., Adson, D., Kotlyar, M., ... \& Foa, E. B. (2007). D-cycloserine augmented exposure therapy for obsessive-compulsive disorder. Biological Psychiatry, 62(8), 835-838. doi:10.1016/j.biopsych.2006.12.020

Mataix-Cols, D., de la Cruz, L. F., Monzani, B., Rosenfield, D., Andersson, E., Pérez-Vigil, A., ... \& Farrell, L. J. (2017). D-Cycloserine augmentation of exposure-based cognitive behavior therapy for anxiety, obsessive-compulsive, and posttraumatic 
stress disorders: A systematic review and meta-analysis of individual participant data. Jama Psychiatry, 74, 501-510.

Mathews, A., \& Mackintosh, B. (2000). Induced emotional interpretation bias and anxiety. Journal of Abnormal Psychology, 109(4), 602. doi: 10.1037/0021843X.109.4.602

Moss, A., Curran, H. V., Bloomfield, M. A. P., Kamboj, S. K., Blackwell, S. E., \& Freeman, T. P. (2016). Bringing together pharmacological and psychological approaches to mental health research. Lancet Psychiatry, 3, 700-702, doi: 10.1016/S22150366(16)30080-3

Patel, D. S., Sharma, N., Patel, M. C., Patel, B. N., Shrivastav, P. S., \& Sanyal, M. (2011). Development and validation of a selective and sensitive LC-MS/MS method for determination of cycloserine in human plasma: application to bioequivalence study. Journal of Chromatography B, 879(23), 2265-2273.

Onur, O. A., Schlaepfer, T. E., Kukolja, J., Bauer, A., Jeung, H., Patin, A., ... \& Fink, G. R. (2010). The N-methyl-D-aspartate receptor co-agonist D-cycloserine facilitates declarative learning and hippocampal activity in humans. Biological Psychiatry, 67(12), 1205-1211.

Otto, M. W., Kredlow, M. A., Smits, J. J., Hofmann, S. G., Tolin, D. F., de Kleine, R. A., \& ... Pollack, M. H. (2016). Enhancement of psychosocial treatment with d-cycloserine: Models, moderators, and future directions. Biological Psychiatry, 80(4), 274-283. doi:10.1016/j.biopsych.2015.09.007

Otto, M. W., Tolin, D. F., Simon, N. M., Pearlson, G. D., Basden, S., Meunier, S. A., ... \& Pollack, M. H. (2010). Efficacy of d-cycloserine for enhancing response to cognitivebehavior therapy for panic disorder. Biological Psychiatry, 67(4), 365-370. doi: 10.1016/j.biopsych.2009.07.036 
Reinecke, A., \& Harmer, C. (2014). A cognitive-neuropsychological account of treatment action in anxiety: Can we augment clinical efficacy. Psychopathology Review.

Rothbaum, B. O., Price, M., Jovanovic, T., Norrholm, S. D., Gerardi, M., Dunlop, B....., et al. (2014): A randomized, double-blind evaluation of Dcycloserine. or alprazolam combined with virtual reality exposure therapy for posttraumatic stress disorder in Iraq and Afghanistan War veterans. American Journal of Psychiatry, 171, 640-648.

Santa Maria, A., Reichert, F., Hummel, S. B., \& Ehring, T. (2012). Effects of rumination on intrusive memories: does processing mode matter? Journal of Behavior Therapy and Experimental Psychiatry, 43(3), 901-909. doi: 10.1016/j.jbtep.2012.01.004

Schartau, P. E., Dalgleish, T., \& Dunn, B. D. (2009). Seeing the bigger picture: training in perspective broadening reduces self-reported affect and psychophysiological response to distressing films and autobiographical memories. Journal of Abnormal Psychology, 118(1), 15. doi: 10.1037/a0012906

Scholl, J., Günthner, J., Kolling, N., Favaron, E., Rushworth, M. F., Harmer, C. J., \& Reinecke, A. (2014). A role beyond learning for NMDA receptors in reward-based decision-making a pharmacological study using D-cycloserine. Neuropsychopharmacology, 39(12), 2900-2909. doi: 10.1038/npp.2014.144

Spielberger, C. D., Gorsuch, R. L., Lushene, R., Vagg, P. R., \& Jacobs, G. A. (1983). Manual for State-Trait Anxiety Inventory. Palo Alto, CA: Consulting Psychologists Press.

Sotres-Bayon F, Bush DE, LeDoux JE (2007): Acquisition of fear extinction requires activation of NR2B-containing NMDA receptors in the lateral amygdala. Neuropsychopharmacology, 32, 1929-1940.

van Berckel, B. N., Lipsch, C., Timp, S., Gispen-de Wied, C., Wynne, H., Van Ree, J. M., \& 
Kahn, R. S. (1997). Behavioral and neuroendocrine effects of the partial NMDA agonist D-cycloserine in healthy subjects. Neuropsychopharmacology, 16(5), 317324.

Weiss, D. S., \& Marmar, C. R. (1997). The Impact of Event Scale - Revised. Assessing Psychological Trauma and PTSD: A Handbook for Practitioners, 399-411.

Woud, M. L., \& Becker, E. S. (2014). Editorial for the special issue on cognitive bias modification techniques: An introduction to a time traveller's tale. Cognitive Therapy and Research, 38, 1-6.

Woud, M. L., Holmes, E. A., Postma, P., Dalgleish, T., \& Mackintosh, B. (2012). Ameliorating intrusive memories of distressing experiences using computerized reappraisal training. Emotion, 12(4), 778-784. doi:10.1037/a0024992

Woud, M. L., Postma, P., Holmes, E. A., \& Mackintosh, B. (2013). Reducing analogue trauma symptoms by computerized reappraisal training - Considering a cognitive prophlaxis? Journal of Behavior Therapy and Experimental Psychiatry, 44(3), 312315. doi:10.1016/j.jbtep.2013.01.003

Woud, M.L., Verwoerd, J.R.L., \& Krans, J. (2017). Modification of cognitive biases related to posttraumatic stress: A systematic review and research agenda. Clinical Psychology Review, 54, 81-95. 


\section{Figure captions}

Figure 1. Flowchart of experimental procedure. Times in the right hand column represent time since administration of placebo/DCS.

Figure 2. Mean difference scores mood and distress pre-post CBM. Mood change pre CBM: Mood II / Mood after 1st recall - Mood I / mood before 1st recall; Mood changes post CBM: Mood IV / Mood after 2nd recall - Mood III / Mood before 2nd recall; Distress change:

Distress post CBM-App - Distress pre CBM-App. Error bars represent standard deviations. 
DCS in emotional learning

Woud et al.

\section{Footnote}

1. The paper reporting these data is currently in preparation.

2. Due to missing data of one participant of the placebo group the $\mathrm{N}$ for both ERTs is $\mathrm{N}=18$. 